\title{
Biological and histopathological characteristics of heat and biochemical processed Kariya (Hildegardia bateri) seeds
}

\author{
Abiodun Victor Ikujenlola ${ }^{1 *}$, Olamide Esther Aluko ${ }^{1}$, Saka Olasunkanmi Gbadamosi ${ }^{1}$ and David Olawale Adeyemi ${ }^{2}$ \\ ${ }^{1}$ Department of Food Science and Technology, Obafemi Awolowo University, Ile-Ife, Nigeria \\ ${ }^{2}$ Department of Anatomy and Cell Biology, Obafemi Awolowo University, Ile-Ife, Nigeria
}

\begin{abstract}
This study assessed the nutritional and safety qualities of kariya seeds subjected to heating and biochemical processes using animal feeding experiment. The kariya seeds were cleaned and processed into flour samples. Diets containing 20\% processed kariya seed flour samples and $80 \%$ vitamin A maize flour samples were produced and fed to healthy wistar rat. Parameters monitored included feed intake, growth performance, mortality rate, biochemical and histopathological tests using standard methods. Data obtained were analyzed using appropriate descriptive and inferential statistics. The results showed that the kariya based diets and basal diet did not support growth of the experimental animals and 100\% mortality was recorded for the heat processed samples while the kariya seeds subjected to biochemical processes (Defatted germinated kariya diet, defatted fermented kariya diet, defatted germinated-fermented kariya diets and germinated whole flour kariya diet) recorded 80, 80, 40 and $80 \%$ mortality respectively. The liver of the rats degenerated in some of the rats fed with heat treated kariya seed flour samples.
\end{abstract}

In conclusion, the biochemical processes detoxified kariya seed better than the heat treatment processes. The flour from kariya seed subjected to biochemical processes recorded lower mortality rate and no liver degeneration in experimental animals.

\section{Introduction}

Agricultural produce contain some undesirable components which are often injurious to the health of consumers; processing is one of the methods used to inactivate or remove any undesirable components. Several processes including fermentation, germination, cooking, boiling, roasting, etc. have been identified as means of reducing, removing and detoxifying contaminants from biomaterials. Fermentation is defined as a desirable biochemical process or biochemical changes caused by microorganisms and their enzymes to desirable products during processing [1]. It devices a natural way to reduce volume of material to be transported, to destroy undesirable component, to enhance nutritive value and appearance of food, to reduce cooking time and energy and to obtain a safe food product [2]. Germination is a natural biological process of all superior plants by which the seed comes out of its latency stage. The process of germination has been developed in some countries as an alternative to reduce some of disadvantages associated with untreated grains, such as undesirable taste and smells, as well as the presence of trypsin inhibitors and phytates [3].The most important physiological processes associated with the germination phase are the synthesis of amylases, proteases and other endogenous hydrolytic enzymes [4]. Cooking has often been considered as means to improve the texture, palatability and nutritive value of cereal due to gelatinization of starch, denaturation of proteins, increased nutrient availability and inactivation of heat labile toxic compounds [5]. Detoxification of potentially toxic chemicals, drugs and environmental contaminants is mainly related to the function of an applicable and healthy liver [6]. The hepatic gland performs a strategetic role in the digestive process by receiving the nutrients from the diet and orchestrating their transformation into useful biomolecules to be delivered to organs and tissues. Liver function tests are carried out to investigate hepatic integrity, and the synthetic, storage, transport, secretory, and other functions of this important organ. Measurement of aspartate transaminase (AST) and alanine transaminase (ALT) are two of the common tests. The serum activity presumably increases as a result of cellular membrane damage and leakage [7]. Histopathology is the study of microscopic changes or abnormalities in tissues that are caused as a result of diseases. Histology is the study of the microscopic anatomy (microanatomy) of cells and tissues of plant and animals. Histological staining is a series of technique processes undertaken in the preparation of sample tissues by staining using histological stains to aid in the microscope study [8]. Previous works on kariya showed that kariya seeds are rich sources of protein (17.5\%) and fat (37.5\%) [9]. However, recent works on kariya seeds have implicated the seed in the death of experimental animals. This has raised serious concern about the safety of the seeds or its product for human consumption [10]. Previous study by Ikujenlola et al. [10] found that the kariya seeds contain some inherent deleterious compounds which were not properly removed during processing hence the need to investigate the effect of several other processing methods on the safety assessment by carrying out in vivo studies and possibly establish the best processing method for safe consumption of kariya seeds and its products as food and food ingredients. Therefore, the aim of this study was to subject kariya seed

${ }^{*}$ Correspondence to: Ikujenlola AV, Department of Food Science and Technology, Obafemi Awolowo University, Ile-Ife, Nigeria, E-mail: avjenlola@gmail.com

Key words: Aspartate aminotransferase, feed intake, fermentation, germination, liver, mortality rate

Received: August 09, 2020; Accepted: September 04, 2020; Published: September 7, 2020 
to various heat and biochemical treatments and assessing the flour using biological and histopathological tools.

\section{Materials and methods}

Dispersed kariya pods were gathered from ornamental kariya trees in Obafemi Awolowo University, Ile-Ife. The nuts were extracted from the pods and sorted to remove extraneous materials. The kernels were obtained by shelling the nuts manually which were then cleaned to remove chaff, broken, shriveled and immature kernels. All reagents used were of analytical grade and were obtained from Sigma Aldrich chemical company, USA.

\section{Production of processed kariya flour}

Dried kariya kernels were divided into eight portions, one portion served as the control (i.e. unprocessed seed) and each of the next five portions was subjected to different processing treatments: boiling $\left(100^{\circ} \mathrm{C}, 1 \mathrm{~h}\right)$, autoclaving $\left(121^{\circ} \mathrm{C}, 15 \mathrm{psi}, 30 \mathrm{~min}\right)$, roasting $\left(100^{\circ} \mathrm{C}, 1\right.$ h), fermentation $\left(28 \pm 2^{\circ} \mathrm{C}, 96 \mathrm{~h}\right)$ and combination of germination $(28$ $\left.\pm 2^{\circ} \mathrm{C}, 96 \mathrm{~h}\right)$ and fermentation $\left(28 \pm 2^{\circ} \mathrm{C}, 96 \mathrm{~h}\right)$ and the remaining two portions were subjected to germination $\left(28 \pm 2^{\circ} \mathrm{C}, 96 \mathrm{~h}\right)$ treatment. The processed seeds from the five portions were milled into flour and the flour from each processing treatment was defatted. Then the two portions that were germinated were divided into two portions and the first portion was left as whole flour and the second portion was defatted using cold acetone extraction ( $1: 5 \mathrm{w} / \mathrm{v}$ solvent:flour).

\section{Formulation of processed kariya flour based complementary diets}

Complementary diets were produced from blends of maize (Vitamin A maize variety) blended with processed kariya flour according to Ikujenlola et al. [10,12]. Table 1 shows the various formulations. Table 2 shows the composition of the basal diet.

Table 1. Formulation of treatment diet $(\mathrm{g} / \mathrm{kg})$

\begin{tabular}{|c|l|}
\hline Diet & Formulation \\
\hline RKD & $80 \%$ vitamin A maize $+20 \%$ Raw kariya flour \\
\hline DCKB & $80 \%$ vitamin A maize $+20 \%$ Cooked kariya flour \\
\hline DGKD & $80 \%$ vitamin A maize $+20 \%$ Germinated kariya flour \\
\hline DRKD & $80 \%$ vitamin A maize $+20 \%$ Roasted kariya flour \\
\hline DFKD & $80 \%$ vitamin A maize $+20 \%$ Fermented kariya flour \\
\hline DAKD & $80 \%$ vitamin A maize $+20 \%$ Autoclaved kariya flour \\
\hline DGFKD & $80 \%$ vitamin A maize $+20 \%$ Germinated- Fermented kariya flour \\
\hline GKD & $80 \%$ vitamin A maize $+20 \%$ Germinated kariya flour \\
\hline BD & Basal (non-protein) diet \\
\hline CD & Commercial diet (control) \\
\hline AF & Animal feed \\
\hline
\end{tabular}

RKD: Raw kariya Diet; DCKD: Defatted cooked kariya diet; DGKD: Defatted germinated kariya diet; DRKD: Defatted roasted kariya diet DFKD: Defatted fermented kariya diet; DAKD: Defatted autoclaved kariya diet DGFKD: Defatted germinated-Fermented kariya diet; GKD: Germinated Whole Flour kariya diet; BD: Basal diet; CD: commercial diet; AF: Animal feed

Table 2. Composition of basal diet

\begin{tabular}{|c|c|}
\hline Component & Quantity (g) \\
\hline Protein & 0 \\
\hline Corn starch & 800 \\
\hline Sugar & 60 \\
\hline Vegetable oil & 120 \\
\hline Vitamin mix & 20 \\
\hline
\end{tabular}

Source: Ikujenlola and Fashakin [13]

\section{Experimental design}

The method of Ikujenlola and Fashakin [13] was adopted. For this study, 55 adult wistar albino rats of both sexes of varying weights were obtained from Animal House, OAU, Ile-Ife, Nigeria. They were distributed into 3 different joint cages based on their sex and then acclimatized for 7 days on grower mash pellet and water was given adlibitum. The animals were weighed and divided into 11 groups with the initial average weight per group and they were distributed into labeled individual metabolic cages. Groups 1-8 were fed with the various diets containing $80 \%$ vitamin A maize and $20 \%$ treated Kariya seed flour while group 9 and group 10 were fed with negative control diet (basal diet) and positive control diet (commercial diet), respectively and group 11 were fed with grower mash feed. Water was provided adlibitum with the feed for a period of 28 days. During the period, two days interval measurement of weight were recorded and feed intake was recorded daily to measure growth rate.

\section{Histopathological study}

After the period of 28 days, the rats were anaesthetized under light ether anesthesia and blood samples were collected by cardiac puncture into plain bottles. The liver was excised, weighed and the right and median lobes were separated. The right lobe was refrigerated (at $4 \mathrm{oC}$ ) in a sterile container while the median lobe was fixed in $10 \%$ neutral buffered formalin for 48 hours for histopathological study. The fixed liver samples were processed via paraffin wax embedding method, sectioned at $5 \mu \mathrm{m}$ thickness and stained by hematoxylin and eosin, Massons trichrome stain, periodic acid schiff (PAS) and Gordon and Sweets silver stain for the assessment of the general histoarchitecture, collagen fibres, glycogen and reticulin fibres of the liver respectively. the stained section were examined on a Leica DM 750 research microscope connected to Leica ICC 50 HD digital camera and digital photomicrographs were taken at various magnifications.

\section{Biochemical assay}

The blood samples were allowed to coagulate and were centrifuged at $3000 \mathrm{x} \mathrm{g}$ for $10 \mathrm{~min}$. Serum samples were separated and stored at 4 ${ }^{\circ} \mathrm{C}$ in a refrigerator. The right lobe of the liver was also homonenized in phosphate buffered saline $(10 \% \mathrm{w} / \mathrm{v})$. the homogenate was centrifuged at $6000 \mathrm{x} \mathrm{g}$ for $15 \mathrm{~min}$. and the supernatant was separated and stored in a refrigerator. Alanine amino transferase (ALT) and aspartate amino transferase (AST) were measured spectrophotometrically in serum and liver homogenate using enzyme colorimetric assay kits (Randox, Northern Ireland) using standard methods.

\section{Statistical analysis}

All determinations were made in triplicates and the data generated were subjected to one-way analysis of variance at $5 \%$ level of significance using SPSS 20.0 for Windows. Means were separated by Duncan's multiple range tests.

\section{Results and discussion}

\section{Feed intake and growth rate of experimental animals}

The feed intake (224.00 g) of the animals fed (Table 3) with commercial diet (CD) and animal feed (AF) which served as controls were appreciably higher than the other groups fed with kariya based diets. It was observed that animals placed on kariya based diets recorded low feed intake and there were variations in the feed intake among the groups. The high feed intake and low feed intake of the controls and 
the kariya based diet respectively showed the level of acceptability and palatability of the diets and by implication the support/sustenance of the diets to the life of the experimental animals. This observation confirms the report of Jansman and Longstaff [14] that the toxic effects of tannins could be categorized as: depression of food intake, inhibition of digestive enzymes, increased excretion of endogenous protein. It was observed that there was positive and negative deviation from the initial overall mean weight of the animals which was $109.54 \pm 2 \mathrm{~g}$ at initial stage of the experiment to around $61.20 \mathrm{~g}$ to $149.76 \mathrm{~g}$ at the end of the experimental period. Weight loss was observed from the $4^{\text {th }}$ day into the feeding trial with the animals fed on the experimental diet and basal diet while gradual increase in weight was observed in the animals fed on commercial diet throughout the feeding trial and insignificant weight gain or loss was observed in the animals fed on animal feed at the early days of the trial but as the feeding trial progressed, significant weight gain was recorded but not as much as that of the group fed with commercial diet. It was observed that the commercial diet and animal feed supported growth positively but animals fed with commercial diet had higher weight compared to those fed with animal feed. The experimental diets which were the processed kariya seed flour and basal diet did not support growth there was weight loss recorded throughout the experimental period. Most times, weight gain or loss could be attributed to the feed intake of the animals and as shown in Table 3 and Figure 1, it was observed that weight gain or loss is a function of feed intake. The animals that consumed large quantity of feed gained more weight while those that consumed small quantity, suffered loss in weight which implies that the small quantity of feed consumed could only support their metabolic activities and not their body growth.

According to previous studies on kariya by Inglet et al. [15]; Ogunsina et al. [9]; Adebayo et al. [16] and Ikujenlola et al. [10], the nutritional composition of kariya has crude protein of about 17.5-22.16 $\mathrm{g} / 100 \mathrm{~g}$ in which should be enough to meet the protein requirement of the experimental animals, therefore it might be right to assumed that palatability of the treated diets and antinutritional factors inherent in kariya seed might be responsible for the low feed intake which resulted into decline in weight gain and which might as well be responsible for the high mortality rate recorded for the animals fed on the experimented diet. Moreover, the decline in growth rate recorded for the animals fed with basal diet could be attributed to deficiency of protein in basal diet and this was the reason for the decline in growth. Similar observation was reported by Ikujenlola et al. [10] for basal diet. This agrees with the observations reported by De Silva, [17] and Alais and Linden [18] that proteins are essential parts of the animals' diet, since they cannot synthesize all the amino acids, they must obtain these essential amino acids from food.

\section{Mortality rate of experimental animals during the feeding trial}

The mortality rate of the experimental animals during the feeding trial is presented in Figure 2. There was no mortality recorded in groups of animal fed with basal diet, commercial diet and animal feed. However,

Table 3. Feed intake of experimental animals during feeding trial

\begin{tabular}{|c|c|c|c|c|c|c|c|c|c|c|c|}
\hline \multirow{2}{*}{ Feed intake } & \multicolumn{11}{|c|}{ Dietary groups of animals } \\
\hline & RKD & DCKD & DGKD & DRKD & DFKD & DAKD & DGFKD & GKD & BD & CD & AF \\
\hline \begin{tabular}{|c|} 
Total Feed \\
Intake/ rat $/ 28$ \\
days \\
\end{tabular} & 70.71 & 120.20 & 141.41 & 123.57 & 129.79 & 90.42 & 122.03 & 114.89 & 156.40 & 224.00 & 224.00 \\
\hline $\begin{array}{l}\text { Mean feed } \\
\text { intake/ rat }\end{array}$ & 3.72 & 5.72 & 5.05 & 6.18 & 4.81 & 5.65 & 4.36 & 4.10 & 5.59 & 8.00 & 8.00 \\
\hline
\end{tabular}

RKD: Raw kariya Diet; DCKD: Defatted cooked kariya diet; DGKD: Defatted germinated kariya diet; DRKD: Defatted roasted kariya diet DFKD: Defatted fermented kariya diet; DAKD: Defatted autoclaved kariya diet DGFKD: Defatted germinated-Fermented kariya diet; GKD: Germinated Whole Flour kariya diet; BD: Basal diet; CD: commercial diet; AF: Animal feed

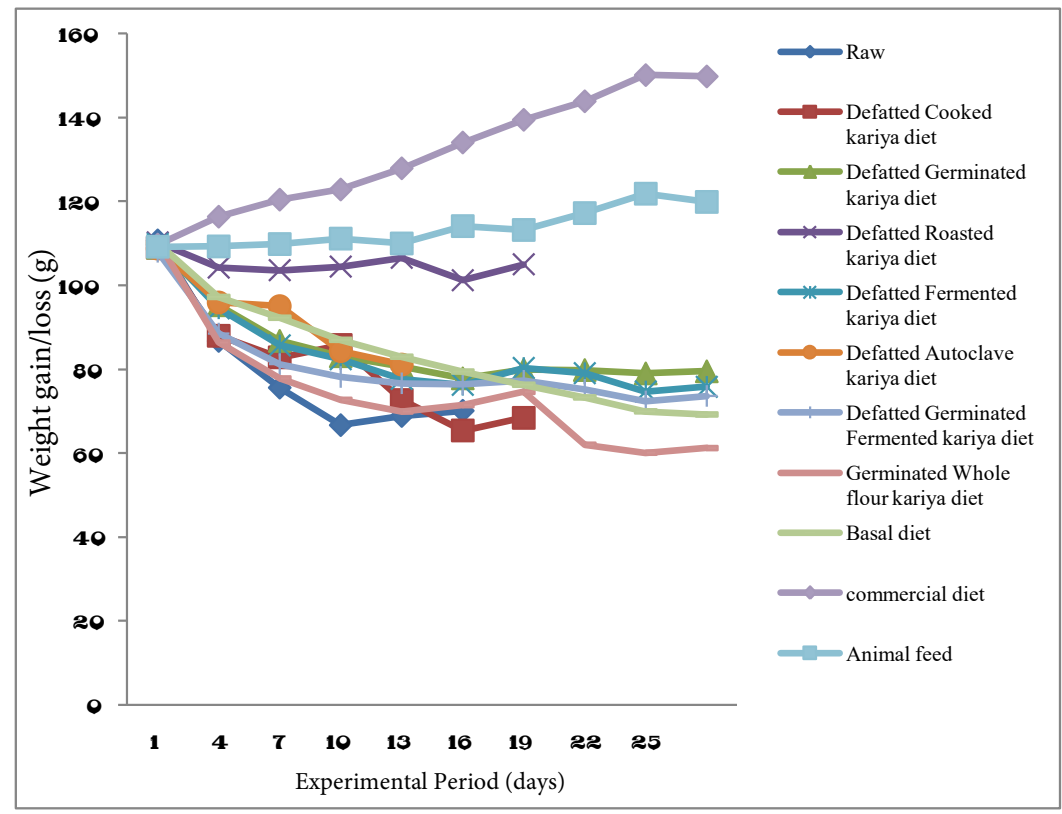

Figure 1. Growth rate of the experimental animals fed with the formulated diets and controls 
mortality at varying percentages was recorded for animals in the diet groups containing raw and processed kariya. It was observed that $\geq$ $20 \%$ mortality was recorded for all the animals fed with kariya based diets and an average of $20 \%$ death was recorded per week. Animals fed with diets RaKD, DAKD, DCKD and DRKD died at the end of the (third week and fourth week) of the feeding trial. Ikujenlola et al. [10] reported that all the animals fed with kariya concentrate blends died after two weeks of feeding trial. However, this study recorded $100 \%$ mortality for the aforementioned four groups at the third and fourth week of the experimental period and the remaining four groups (DGKD, DFKD, DGFKD and GKD) fed with the treated kariya diet had survivors till the end of the experimental period and their mortality rate were recorded as follows $(80,80,40$, and $80 \%)$ respectively. This result implied that the processing treatments has improved the safety status of kariya meal and combination of germination and fermentation treatments showed more promising prospect that if the dose and treatments are well harmonized, kariya based diet could become safer to the consumer.

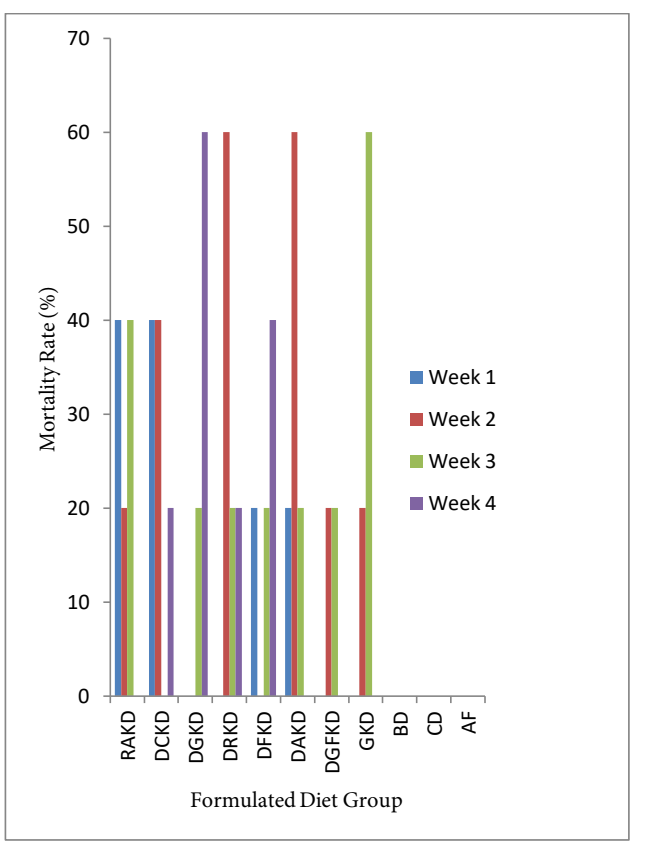

Figure 2. Mortality rate during the experimental period. RKD: Raw kariya diet; DCKD: Defatted cooked kariya diet; DGKD: Defatted germinated kariya diet; DRKD: Defatted roasted kariya diet DFKD: Defatted fermented kariya diet; DAKD: Defatted autoclaved kariya diet DGFKD: Defatted germinated-Fermented kariya diet; GKD: Germinated Whole Flour kariya diet; BD: Basal diet; CD: commercial diet; AF: Animal feed

Table 4. AST and ALT levels $(\mathrm{U} / \mathrm{L})$ in the liver and serum of wistar albino rats fed with treated kariya seed flour meal

\begin{tabular}{|c|c|c|c|c|}
\hline \multirow{2}{*}{ Sample } & \multicolumn{2}{|c|}{$\begin{array}{c}\text { Aspartate Transaminase (AST) } \\
(\mathbf{U} / \mathbf{L})\end{array}$} & \multicolumn{2}{|c|}{$\begin{array}{c}\text { Alanine Transaminase (ALT) } \\
(\mathbf{U} / \mathbf{L})\end{array}$} \\
\cline { 2 - 5 } & Liver & Serum & Liver & Serum \\
\hline DGKD & $326.44 \pm 30.35^{\mathrm{a}}$ & $79.85 \pm 11.36^{\mathrm{a}}$ & $251.41 \pm 8.6^{\mathrm{a}}$ & $67.14 \pm 13.33^{\mathrm{b}}$ \\
\hline DFKD & $102.09 \pm 14.83^{\mathrm{e}}$ & $43.60 \pm 5.67^{\mathrm{bc}}$ & $223.38 \pm 6.57^{\mathrm{bc}}$ & $36.70 \pm 11.61^{\mathrm{c}}$ \\
\hline DGFKD & $165.68 \pm 18.52^{\mathrm{d}}$ & $55.13 \pm 13.52^{\mathrm{b}}$ & $217.10 \pm 14.15^{\mathrm{c}}$ & $71.03 \pm 13.75^{\mathrm{a}}$ \\
\hline GKD & $195.27 \pm 8.00^{\mathrm{bc}}$ & $14.77 \pm 0.83^{\mathrm{d}}$ & $217.49 \pm 6.67^{\mathrm{c}}$ & $53.04 \pm 3.70^{\mathrm{bc}}$ \\
\hline BD & $245.10 \pm 19.00^{\mathrm{b}}$ & $54.23 \pm 6.15^{\mathrm{b}}$ & $259.26 \pm 5.73^{\mathrm{a}}$ & $57.11 \pm 3.19^{\mathrm{ab}}$ \\
\hline CD & $167.15 \pm 15.56^{\mathrm{d}}$ & $13.15 \pm 1.60^{\mathrm{dc}}$ & $254.97 \pm 3.91^{\mathrm{a}}$ & $55.03 \pm 0.35^{\mathrm{ab}}$ \\
\hline AF & $213.76 \pm 5.16^{\mathrm{c}}$ & $31.82 \pm 6.33^{\mathrm{c}}$ & $234.61 \pm 2.24^{\mathrm{b}}$ & $49.68 \pm 8.80^{\mathrm{bc}}$ \\
\hline
\end{tabular}

Values with different superscript down the group are significantly different at $\mathrm{P}<0.05$ DGKD: Defatted cooked kariya diet; DFKD: Defatted fermented kariya diet; DGFKD: Defatted germinated-fermented kariya diet; GKD: Germinated whole flour kariya diet; BD: Basal diet; CD: Commercial diet; AF: Animal feed

\section{Liver function test of experimental animals}

Biochemical indices of liver function test using Alanine aminotransferase (ALT) and Aspartate aminotransferase (AST) as enzyme marker: The normal ranges of ALT and AST in the serum are 10-55 $\mathrm{UL}^{-1}$ and $10-40 \mathrm{UL}^{-1}$ respectively and the measure of degree of damage such as mild, moderate and severe damage of the liver are 1-3 times, 3-20 times and $>20$ times or $1000 \mathrm{UL}^{-1}$ of the normal ALT and AST level [19]. Using this biochemical indices for this study and considering the values recorded in Table 4 which is showing the serum and liver AST and ALT level of the experimental animals after the feeding trial, it was observed that the liver- ALT and AST levels of the animals in all the diet groups are much higher than the serum-ALT and AST levels. According to Thapa and Walia [19] this trend is expected under normal condition except when there is liver damage, also ALT level is usually more frequently increased as compared to AST level except in chronic liver damage and this agrees with the values recorded for all the processed kariya diets and control except diet DGKD which the AST level of the liver was higher than that of the ALT level. This implied cellular damage to the plasma membrane and it is an indication that the diet might not be safe for consumption.

From the study, the enzyme activity in the serum of animals fed with diets DGKD, DGFKD and BD were above the normal range of ALT and AST levels but they are within the mild level of liver damage because their activity was between 1-3 times of the normal ALT and AST levels. Also there was significant increase $(p<0.05)$ in the serum enzyme activity of the animals fed with the diets compared to the control diet and this implied cellular damage to the plasma membrane of the animals' organs. Moreover, Ashafa et al. [20] reported that ALT is regarded to be more specific indicator of liver inflammation because hyper AST may be caused by other diseases of other organs such as heart and muscles. Based on the biochemical indices, it was observed that animals fed with diets DGKD and DGFKD had abnormal range of ALT level in their serum and the damage was mild but the serum level of animals fed with diets DFKD, GKD, BD, CD, and AF are within the normal range.

Effect of AST: ALT ratio on liver damage of experimental animals: The AST: ALT ratio of the experimental animals which shows the level of liver damage is presented in Table 5. AST: ALT ratio provides useful clinical information regarding both the cause and severity of liver disease [21]. When the AST: ALT $\geq 2$ it indicates a chronic damage of the liver whereas if AST: ALT $\geq 1$ it indicates an acute hepatitis/liver injury. Using this biochemical indices, in this study, it was observed that animals that were fed with diets DGKD and DFKD had AST: ALT $\geq 1$ and this implied acute hepatitis or liver injury which may heal up with time [19].

\section{Organ-to-body weight ratio of experimental animals}

Table 6 shows the organ to body weight ratio. Alteration in organto-body weight ratio may be as a result of organ damage [22]. The mean organ-to-body weight ratio evaluated were not significantly different $(\mathrm{P}>0.05)$ from the control, that is, the organ to body weight ratios were not significantly affected in the test rats compared with the control. This implied that the use of liver to body weight ratio as a biochemical indices did not reveal the liver status in this study, however histopathological study gave clearer information on the liver status. 


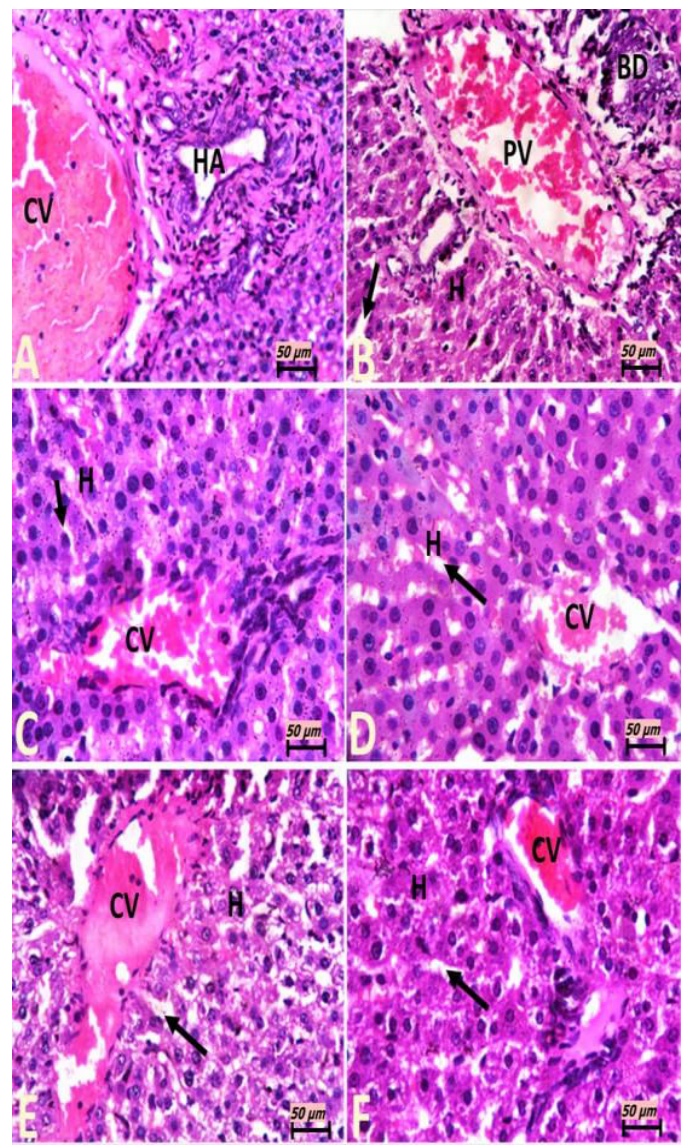

Figure 3. Photomicrographs showing liver sections of experimental rats (A- F) stained with Hematoxylin and Eosin stain. Note plates of hepatocyte $(\mathrm{H})$ radially located around the central vein (CV) with sinusoids (black arrow) between them. Also observe the portal vein (PV), hepatic artery (HA) and bile duct (BD). Scale bar $=50 \mu \mathrm{m}$. A: Defatted germinated kariya diet, B: Defatted fermented kariya diet, C: Defatted germinated-fermented kariya diet, D: Germinated whole flour kariya diet, E: Basal diet, F: Commercial diet (Control)

Table 5. AST: ALT ratio in the serum of experimental rat showing severity of liver damage

\begin{tabular}{|c|c|c|c|}
\hline Sample & AST SERUM & ALT SERUM & AST:ALT \\
\hline DGKD & 79.85 & 67.14 & 1.19 \\
\hline DFKD & 43.6 & 36.7 & 1.19 \\
\hline DGFKD & 55.13 & 71.03 & 0.78 \\
\hline GKD & 14.77 & 53.03 & 0.28 \\
\hline BD & 5.23 & 57.11 & 0.09 \\
\hline CD & 13.15 & 55.03 & 0.20 \\
\hline AF & 31.82 & 49.68 & 0.64 \\
\hline
\end{tabular}

DGKD: Defatted cooked kariya diet; DFKD: Defatted fermented kariya diet; DGFKD: Defatted germinated-fermented kariya diet; GKD: Germinated whole flour kariya diet; BD: Basal diet; CD: Commercial diet; AF: Animal feed

Table 6. Organ-to-body weight ratio of experimented animals

\begin{tabular}{|c|c|c|c|}
\hline Sample & Body weight (g) & Liver Size (g) & Liver: body weight \\
\hline DGKD & $79.20 \pm 0.00^{\mathrm{c}}$ & $2.9 \pm 0.00^{\mathrm{c}}$ & 0.036 \\
\hline DFKD & $75.80 \pm 0.00^{\mathrm{c}}$ & $2.3 \pm 0.00^{\mathrm{de}}$ & 0.030 \\
\hline DGFKD & $73.60 \pm 5.20^{\mathrm{c}}$ & $2.57 \pm 0.25^{\mathrm{cd}}$ & 0.034 \\
\hline GKD & $61.20 \pm 0.00^{\mathrm{d}}$ & $2.10 \pm 0.00^{\mathrm{e}}$ & 0.034 \\
\hline BD & $62.43 \pm 0.97^{\mathrm{d}}$ & $2.2 \pm 0.20^{\mathrm{de}}$ & 0.035 \\
\hline CD & $137.30 \pm 6.97^{\mathrm{a}}$ & $4.47 \pm 0.41^{\mathrm{a}}$ & 0.032 \\
\hline AF & $111.37 \pm 3.00^{\mathrm{b}}$ & $3.43 \pm 0.30^{\mathrm{b}}$ & 0.030 \\
\hline
\end{tabular}

Values with different superscript down the group are different significantly at $\mathrm{P}<0.05$ DGKD: Defatted cooked kariya diet; DFKD: Defatted fermented kariya diet; DGFKD Defatted germinated-fermented kariya diet; GKD: Germinated whole flour kariya diet; BD: Basal diet; CD: Commercial diet; AF: Animal feed

\section{Histopathological examination of liver sections of experimental animals}

The biochemical findings were confirmed by histological observations, which include hepatocellular necrosis, fatty accumulation, tissue fibrosis and other histological alterations of liver of the experimental animals.

Hematoxylin and eosin stain: The photomicrographs of histological examination of liver section of the experimental rat fed with processed kariya diets and controls which were stained with heamatoxylin and eosin (H\&E) stain are shown in Figure 3. The liver cells are arranged as plates of hepatocytes $(\mathrm{H})$ around a central vein $(\mathrm{CV})$ and portal area consisting of portal vein (PV), hepatic artery (HA), bile duct (BD), sinusoids (arrows) between them. Some elements of degeneration of the liver cells were observed in groups A and B but groups C, D and E appeared normal.

Animals fed with defatted germinated kariya diet and defatted fermented kariya diet which were groups A and B respectively, had some elements of liver cells degeneration (hepatic necrosis) while groups C, D, E and F presented normal hepatic plates (shown by arrows between the sinosoids) i.e normal lobular architecture. The necrosis of the liver tissues observed could be attributed to the excessive activity of the experimental animals to get rid of the toxicant from its body during the process of detoxification. Similar observation was reported by Hussien [23] for aqueous extract of Glycyrrhiza glabrous fed to experimental animals. However, Patel and Bahadur [24] suggested that liver incapability in regenerating new cells may lead to necrosis.

Masson trichome stain: The photomicrographs of histological examination of liver section of the experimental rat fed with processed kariya diets and controls which were stained with Masson Trichome stain are shown in Figure 4. The collagen deposition (green stained area) around the central vein, periportal area as well as the intercellular matrix around the hepatocyte was noted. The micrograph revealed over expression of collagen in the liver of group D experimental rats which represents the micrograph of animals fed with undefatted germinated kariya diet and mild collagen deposition was observed on the micrographs of groups A, B, C, E and F. Masson Trichome stain revealed the collagen deposition with a highlight of bluish green colour showing the collagen-rich fibrotic regions which aids in visualizing the extent of fibrosis in the tissue. The over expression of collagen observed in the micrograph of experimental animal fed with undefatted germinated kariya diet could be attributed to the effect of the deleterious constituent in kariya seed on lipid metabolism of liver and this is evident in sample $\mathrm{D}$ because their diet contained high fat content unlike other kariya diet samples fed to groups A, B and C which were defatted samples. This could be attributed to the reduction of lipase activity which could lead to decrease in glyceride hydrolysis [25]. However, Havel [26] suggested that it could result from damage of hepatic parenchyma cells that lead to disturbance of lipid metabolism in liver. Muller et al. [27] and Althnaian et al. [28] reported that carbon tetrachloride $\left(\mathrm{CCl}_{4}\right)$ intoxication is similar to hepatitis in case of the triglyceride catabolism.

Periodic acid-schiff (PAS) stain: The photomicrographs of histological examination of liver section of experimental rat fed with processed kariya diets and controls which were stained with Periodic Acid-Schiff (PAS) stain are shown in Figure 5. PAS stain highlighted a well demonstrated glycogen in all sections of the micrographs of all the groups of experimental animals. According to Diaz-Munoz et al. [29], glycogen staining intensity remained mostly constant in the groups of 


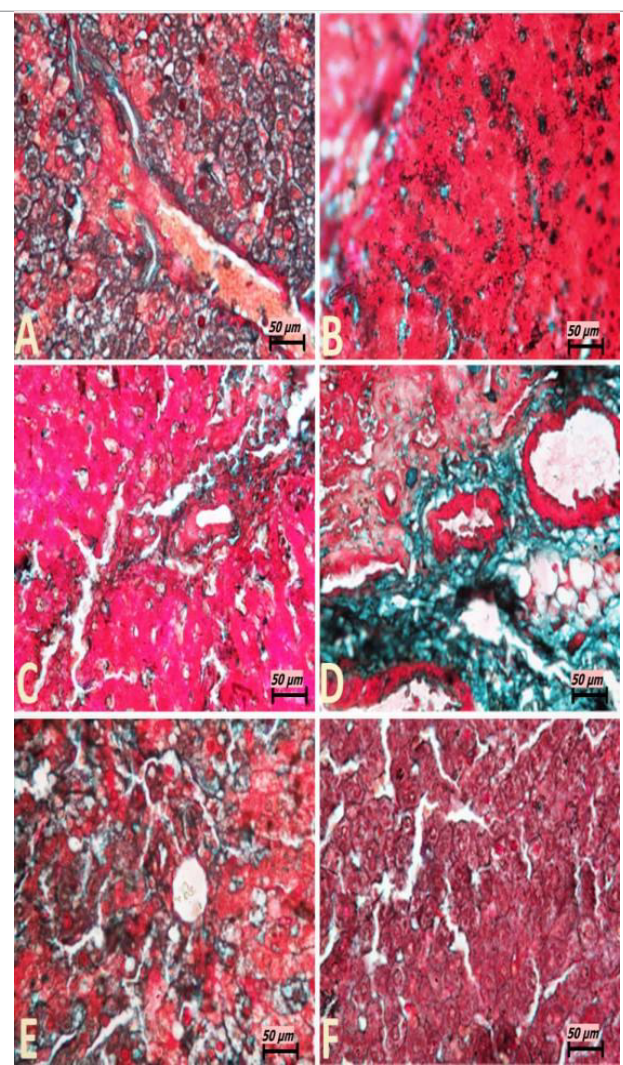

Figure 4. Photomicrographs showing liver sections of experimental rats (A- F) stained with Masson Trichrome stain. Scale bar $=50 \mu \mathrm{m}$. A: Defatted germinated kariya diet; B: Defatted fermented kariya diet; C: Defatted germinated-fermented kariya diet; D: Germinated whole flour kariya diet; E: Basal diet; F: Commercial diet (Control)

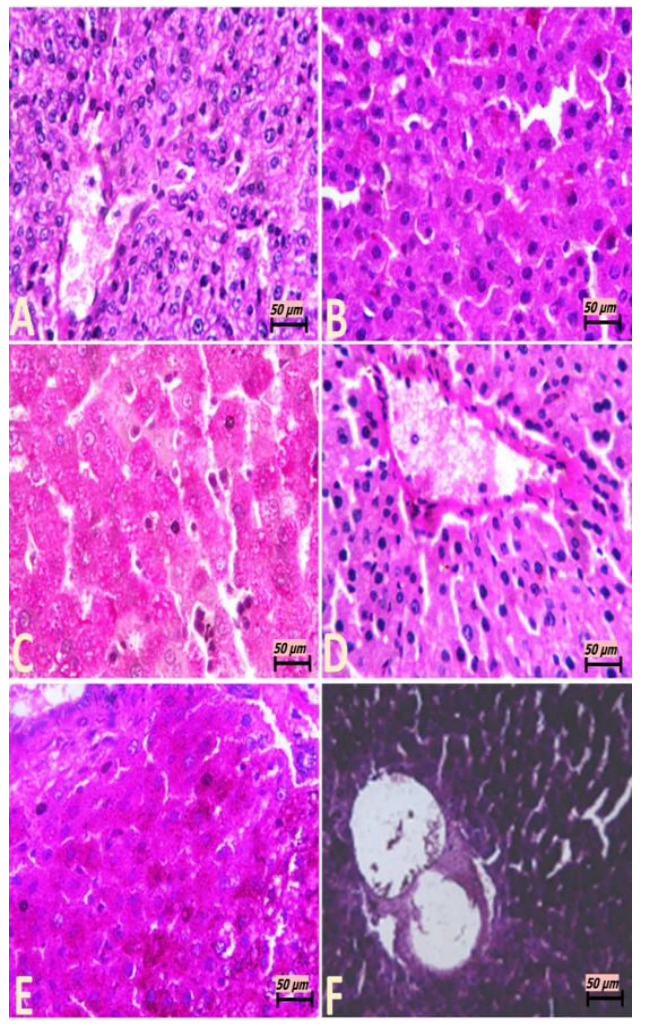

Figure 5. Photomicrographs showing liver sections of experimental rats (A- F) stained with Periodic Acid-Schiff (PAS) stain. Scale bar $=50 \mu \mathrm{m}$. A: Defatted germinated kariya diet; B: Defatted fermented kariya diet; C: Defatted germinated-fermented kariya diet; D: Germinated whole flour kariya diet; E: Basal diet; F: Commercial diet (Control)
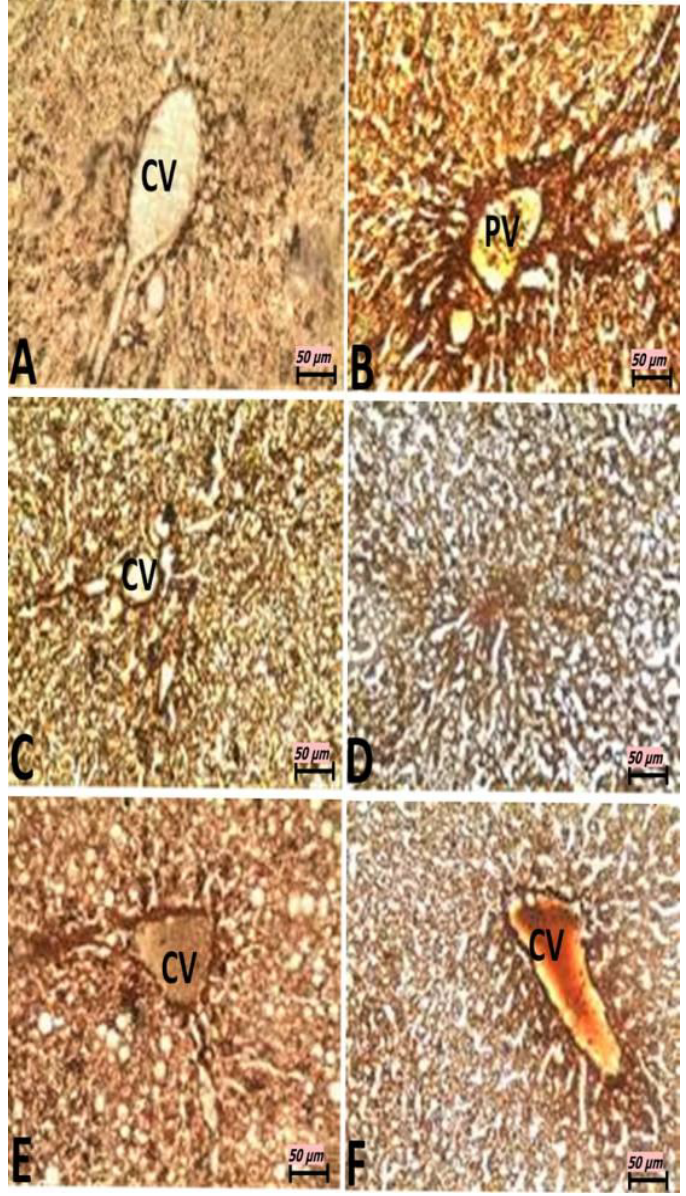

Figure 6. Photomicrographs showing liver sections of experimental rats (A- F) stained with Godon and Sweets' silver stain. Scale bar $=50 \mu \mathrm{m} . \mathbf{A}$ : Defatted germinated kariya diet; B: Defatted fermented kariya diet; C: Defatted germinated-fermented kariya diet; D: Germinated whole flour kariya diet; E: Basal diet; F: Commercial diet (Control)

animals fed ad libitum and this agreed with the observation recorded for the PAS staining of liver section of experimental animals in this study. PAS staining is mainly used for staining structures containing a high proportion of carbohydrates such as glycogen, glycoproteins, proteoglycans typically found in connective tissues, mucus and basement membranes. It is often used to stain kidney biopsies, liver biopsies, certain glycogen storage diseases in striated muscles and suspected fungal infections. PAS staining is useful in identifying glycogen and to reveal glycogen storage disorder.

Godon and sweets' stain: The photomicrographs of histological examination of liver section of experimental rat fed with treated kariya diets and controls which were stained with Godon and sweets' stain are shown in Figure 6. Reticular fibres are present in areas stained black, they are well demonstrated across all groups except group A. Godon and sweets' stain highlighted reticular fibres which are well demonstrated across all the groups, they were seen to be well preserved with distinct inter-hepatic and arterial wall distributions in the liver section as revealed on the micrographs except group A, which were fed with defatted germinated kariya diet. Reticular fibres support the body organs like liver spleen and kidney. Abnormal reticular fibre patterns indicate cirrhosis or necrosis of the liver and it may as well indicate the presence of certain tumors. Group A shows areas of compressed and collapsed reticulin fibres and this correspond to area of reticulin degeneration and cell loss respectively. Reticulin fibres act as support to parenchyma and the reticulin stains provides information about the architecture of the organs. When hepatocytes are damaged and 
undergo necrosis, the reticulum fibre surrounding them collapse in the empty space left behind. Areas of reticulin crowding thus indicate focal hepatocyte loss [30]. Large areas of cell necrosis appeared as reticulum collapse and this indicates reduction of rigidity and mechanical strength function of the fibres [31].

The overall histological examination gives an impression that samples A (defatted germinated kariya diet), B (defatted fermented kariya diet), and $\mathrm{D}$ (germinated whole flour kariya diet) caused liver degeneration while sample C (defatted germinated-fermented kariya diet), E (basal diet) and F (Commercial diet (Control)) could be safe because there were no evidence of liver degeneration on the photomicrograph of their different stains.

\section{Conclusion}

The outcomes of the assessment of kariya seed flour produced after subjecting it to series of processing treatments (heat and biochemical processes) showed the importance of detoxifying of kariya seeds to ensure safety. The study concluded that the effect of heat treatments (autoclaving, cooking and roasting) on kariya seeds were not sufficient to detoxify it in order to prevent mortality in the experimental animals while a combination of the biochemical processes (germination and fermentation) recorded better safety status. Also liver degeneration reduced in the rats fed with kariya seeds treated with biochemical processes.

\section{References}

1. Cheftel JC (1995) Review: high-pressure, microbial inactivation and food preservation. International Journal of Food Science Technology 1: 75-90.

2. Food and Agricultural Organization (FAO) (1995) Sorghum and millets in human nutrition. Food and Agriculture Organization of the United Nations, Italy, Rome.

3. Sangronis E, Machado CJ (2007) Influence of germination on the nutritional Quality of Phaseolus vulgaris and Cajanus cajan. Journal of Science and Technology 40: 116120.

4. Hoseney RC (1994) Principles of cereal science and technology. Second edition St. Paul: American Association of cereal chemistry.

5. Uppal V, Bains K (2012) Effect of germination periods and hydrothermal treatments on in vitro protein and starch digestibility of germinated legumes. Journal of Food Science and Technology 49: 184-191.

6. Bechmann LP, Hannivoort RA, Gerken G, Hotamisligil GS, Trauner M, et al. (2012) The interaction of hepatic lipid and glucose metabolism in liver diseases. Journal of Hepatology 56: 952-964.

7. Kaplan MM (1993) Laboratory tests. In: Schiff L, Schiff ER, editors. Diseases of the Liver. 7th ed. Philadelphia, PA: JB Lippincott.

8. Anderson J (2011) An introduction to Routine and special staining. Retrieved on August 18, 2014 from http://www.leicabiosystems.com/pathologyleaders/an-introduction-toroutine-and-special-staining.

9. Ogunsina BS, Olaoye IO, Adegbenjo AO, Babawale BD (2011) Nutritional and physical properties of kariya seeds (Hidergardia bateri). International Agrophysics 25: $97-100$.

10. Ikujenlola AV, Ahmida AF, Gbadamosi OS (2017) Nutritional quality and safety assessment of complementary food produced from acha (Digitaria exilis) flour and kariya (Hildergardia barteri) protein concentrate blends. Journal of Food Chemistry and Nanotechnology 3: 24-30.
11. PAG (1971) Protein advisory group of the United Nation. Guideline No.8. Protein rich mixture for used as weaning foods. New York: Food and Agriculture Organization of the United Nation/World Health Organization/ United Nation Children Funds, pp: 1-7.

12. FAO/WHO (1991) Protein quality evaluation. Report on Joint FAO/WHO Expert Consultation.

13. Ikujenlola AV, Fashakin JB (2005) Bioassay assessment of a complementary diet prepared from vegetable proteins. Journal of Food, Agriculture and Environment 3: 20-22.

14. Jansman AJM, Longstaff M (1993) Nutritional effects of tannins and vicine/convicine in legume seeds. In: Proceedings of the 2nd International Workshop on Antinutritional Factors in Legume Seeds. Recent Advances of Research in Antinutritional Factors in Legume Seeds (eds. A.F.B. Van der Poel, J. Huisman, H.S. Saini). EAAP Publication, Wageningen, The Netherlands, pp: 301-316.

15. Inglett GE, Cavins JF, Spencer GF (1973) Seed compositions of Hildergardia barteri. Economic Botany Journal 27: 128-130.

16. Adebayo WA, Ogunsina BS, Gbadamosi SO (2013) Some physico-chemical and functional properties of kariya (Hildegardia baterii) kernel flours. Ife Journal of Science 15: 477-486.

17. De Silva SS, Shim KF, Khim OA (1990) An evaluation of the method used in digestibility estimation of a dietary ingredient and comparisons on external and internal markers and time of faeces collection in digestibility studies in the fish Oreochomis aureus (Steindachner). Journal of Reproduction, Nutrition and Development 30: 215-226.

18. Alais C, Linden G (1997) Food Biochemistry. Spen. Publisher, Inc, Gaithersbury, Maryland. 1999, pp: 119-129.

19. Thapa BR, Walia A (2007) Liver function tests and their interpretation. Indian Journal of Pediatrics 74: 663-671.

20. Ashafa AOT, Yakubu MT, Grierson DS, Afolayan AJ (2009) Toxicological evaluation of the aqueous extract of Felicia (Muricuta thumb) leaves in wistar rats. African Journal of Biotechnology 8: 949-954.

21. Nsiah K, Dzogbefia VP, Ansong D, Osei AA, Boateng H, et al. (2011) Pattern of AST and ALT changes in relation to hemolysis in sickle cell disease. Clinical Medicine Insights: Blood Disorders 4: 1-9.

22. Busari MB, Muhammad HL, Ogbadoyi EO, Kabiru AY, Sani S, et al. (2015) In vivo evaluation of antidiabetic properties of seed oil of Moringa oleifera. Journal of Applied Life and Science International 2: 160-174.

23. Hussein E (2013) Biochemical and histopathological studies on the liver of rats administrated with different concentrations of aqueous extract of Glycyrrhiza glabrous. Journal of Global Veterinaria 10: 491-495.

24. Patel JM, Bahadur A (2011) Histopathological manifestations of sub lethal toxicity of copper ions in Catla catla. American-Eurasian Journal of Toxicological Science 4: 1-5.

25. Jahn CE, Schaegfetr EJ, Taam LA (1985) Lipoprotein abnormalities in primary biliary cirrhosis association with hepatic lipase inhibition as well as altered cholestero esterification. Journal of Gastroenterology 89: 1266-1278.

26. Havel RJ (1986) Functional activities of hepatic lipoproteins receptors. Annals of Rev Physiology 48: 119-134.

27. Muller P, Fellin R, Lambreacht J (1974) Hypertriglyceridemia secondary to liver disease. European Journal of Clinical Investigation 4: 419-428.

28. Althnaian T, Albokhadaim I, El-Bahr SM (2013) Biochemical and histopathological study in rats intoxicated with carbontetrachloride and treated with camel milk. Springer Plus Journal 2: 57.

29. Díaz-Muñoz M, Vázquez-Martínez O, Aguilar-Roblero R, Escobar C (2000) Anticipatory changes in liver metabolism and entrainment of insulin, glucagon, and corticosterone in food-restricted rats. American Journal of Physiology 279: 2048-2056.

30. Saxena RM (2010) Special stains in interpretation of liver biopsies. Connection Journal 1: 92-103.

31. Muhammed AO, Adeyemo IA, Olutunde OA, Afodun MA, Ajayi AS (2016) Safety of collagen and reticulin fibres in the liver and kidney of broiler chicken fed with Aspergillus niger - hydrolyzed cassava peel meal as carbohydrate source. Journal of Medical Laboratory and Diagnosis 7: 5-12.

Copyright: (C2020 Ikujenlola AV. This is an open-access article distributed under the terms of the Creative Commons Attribution License, which permits unrestricted use, distribution, and reproduction in any medium, provided the original author and source are credited. 